\title{
Current Trends in Hemodialysis Therapy
}

\author{
Syed N. Asad, M.D., F.A.C.P. \\ Stony Brook, New York
}

DOI: http://dx.doi.org/10.5915/22-4-14317

\begin{abstract}
There are approximately 100,000 patients on dialysis in the U.S. Hemodialysis techniques appear to be in a stagnant phase in comparison to other medical achievements made between 1960-1980. However, despite the lack of major breakthroughs in the management of end stage renal disease, a steady improvement in technology has occurred.

This article describes our experience of chronic dialysis patients managed at our center over many years, with emphasis on certain longterm complications associated with dialysis treatment and end stage renal disease. The biochemical abnormalities in these patients included chronic anemia (HCT 24.9\% \pm 4.97 ), secondary hyperparathyroidism, metabolic acidosis and hyperphosphatemia. The majority of our patients received adequate dialysis as assessed by measuring $K T / V$, the dialysis index.

Current strategies in the management of end stage renal disease patients consist of administering correct prescription dialysis $(K T / V)$, parenteral Vitamin $D_{3}\left(1,25\left(\mathrm{OH}_{2}\right) D_{3}\right)$, Erythropoetin $(R-H u E P O)$ and high flux dialysis with bicarbonate dialysate. The future for dialysis patients is promising because $R-H u E P O$ and high flux dialysis techniques will soon be widely available, which will dramatically improve both the patient's physical well-being and rehabilitation potential.
\end{abstract}

Key words: Hemodialysis, divalent ion metabolism, erythropoetin, prescription dialysis, 1,25( $\left.\left.\mathrm{OH}_{2}\right) \mathrm{D}_{3}\right)$, anemia.

There are more than 100,000 patients on chronic maintenance hemodialysis treatment in the United States. Approximately the same number of patients undergo treatment in Europe. Hemodialysis therapy is also now available in most of the developing countries. The incidence of end stage renal disease varies from region to region in the same country. It appears to be related to many factors, such as socioeconomic conditions, availability of medical technology and age. ${ }^{1}$ About $35-40 \%$ of patients on maintenance dialysis are diabetics. Increasingly large number of patients entering dialysis programs are older patients with renal failure of unknown cause, or due to drug toxicity.

Summarized in table 1 are the biochemical values

From the Department of Medicine

State University of New York at Stony Brook, NY

Reprint Requests: Syed N. Asad, M.D., F.A.C.P.

Huntington Artificial Kidney Center

256 Broadway

Huntington Station, NY 11746 of end stage renal disease (ESRD) patients being treated at our dialysis unit in Huntington, N.Y. Despite dialysis treatment three times a week, patients continue to have abnormal blood milieu. There is persistent azotemia, hyperphosphatemia, anemia, hyperparathyroidism, metabolic acidosis and mild hypocalcemia. In addition, these patients have a higher incidence of hypertension and a tendency to premature atherosclerosis. Figure 1 shows the percentage frequency distribution of serum phosphorus levels in selected patients. Figure 2 shows the serum parathyroid hormone levels (intact PTH measured by Nichols method). Figure 3 shows the correlation between serum phosphorus and serum bicarbonate $(p<.05)$, suggesting that high serum phosphorus levels are associated with severe metabolic acidosis.

Rather than provide a broad but superficial overview, the selection of topics in this review has been based largely on the impact that each has had on the care of patients in our hemodialysis program. 
Table 1. Mean Biochemical values in End Stage Renal Disease (ESRD) patients, 1989.

\begin{tabular}{lcccccccc}
\hline & PTH & KT/V & $\begin{array}{c}\text { BUN } \\
\mathbf{m g} \%\end{array}$ & $\begin{array}{c}\text { HCO3 } \\
\mathbf{m M o l e} / \mathbf{L}\end{array}$ & $\begin{array}{c}\text { Ca } \\
\mathbf{m g} \%\end{array}$ & $\begin{array}{c}\text { PO4 } \\
\mathbf{m g} \%\end{array}$ & $\begin{array}{c}\text { Alk Phos } \\
\text { Units/dl }\end{array}$ & $\begin{array}{c}\text { HCT } \\
\text { Percent }\end{array}$ \\
\hline Mean & 291.00 & 1.12 & 87.91 & 15.43 & 9.58 & 6.69 & 137.99 & 24.93 \\
Std. Dev. & 360.00 & 0.27 & 16.61 & 3.08 & 0.99 & 2.00 & 136.00 & 4.97 \\
Number & 107 & 92 & 92 & 92 & 92 & 92 & 92 & 92 \\
\hline
\end{tabular}

For abbreviations see text.

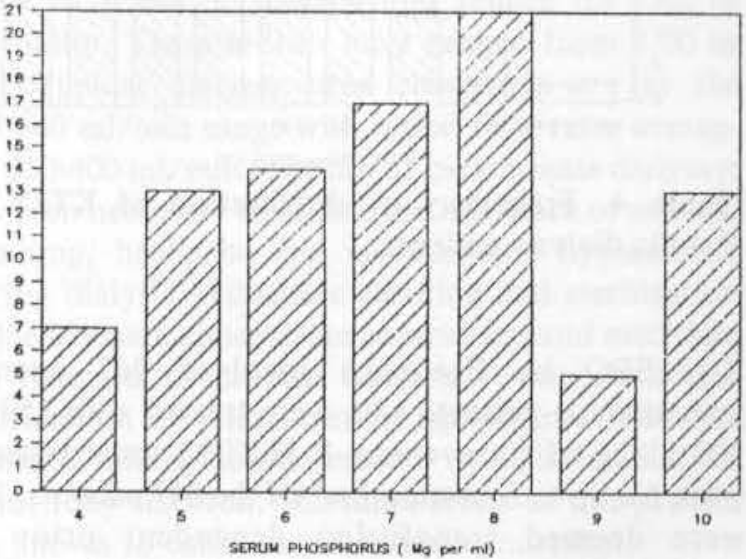

Figure 1. Frequency of distribution of serum phosphorus levels in chronic dialysis patients.

\section{Management strategies}

Management of divalent ion metabolism: Nephrologists are faced with the constant and vexing problem of managing abnormal calcium and phosphorus metabolism in patients undergoing dialysis. ${ }^{2}$ It is desirable to achieve normal calcium levels by administering calcium supplements and oral 1,25 dihydroxy cholecarciferol (Vitamin $\mathrm{D}_{3}$, Rocaltrol by the Roche Company). Now Vitamin $\mathrm{D}_{3}$ is available in the parenteral form (Calcijex made by the Abbott Company) which is given after each dialysis to patients with persistent hypocalcemia and marked secondary hyperparathyroidism. Sufficient control of hyperparathyroidism is now feasible with parenteral Calcijex thus avoiding surgical parathyroidectomy.

Hyperphosphatemia up until recently was treated with aluminum containing binders. But with increasing recognition of alumimum overload in the tissues, with resultant encephalopathy, osteomalacia and microcytic anemia, alumimum containing antacids have been replaced with calcium carbonate. However this requires a reduction in the concentration of calcium in the dialysate fluid. A bone biopsy with histomorphometry provides additional valuable information on patients suffering from aluminum toxicity. We have been successful in optimizing the divalent ion metabolism in the majority of the pa-

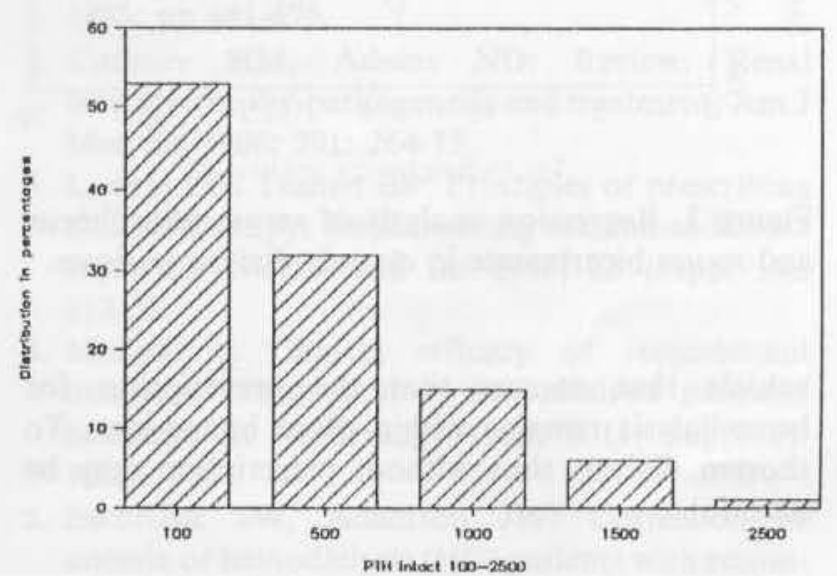

Figure 2. Fequency of distribution of serum parathyroid hormone (PTH) levels in chronic dialysis patients.

tients at our center by judiciously applying these strategies.

\section{Prescription dialysis}

Pressure by patients to limit time on dialysis, constraints on dialysis space and reimbursement and improvement in membrance technology have collectively contributed to the notion that dialysis time may be shortened in a generic manner to most patients. The National Cooperative Dialysis Study (NCDS) has assessed the prescription modality and safety parameters in a large enough population to lend validity to shortening dialysis. ${ }^{3}$ In our center we have done measurements of urea kinetics to compare with various published methods of assessing the adequacy of dialysis by prescription. We have followed the $\mathrm{KT} / \mathrm{V}(\mathrm{K}=$ urea clearance; $\mathrm{T}=$ time on dialysis in minutes; $\mathrm{V}=$ the volume of distribution of urea nitrogen which approximates the total body water), an index of dialysis prescription for all dialysis patients at the center. A value of 1.0 and above is considered optimal and patients with lower values are prescribed either a bigger surface dialyzer or an increased time on dialysis. The majority of our patients have values higher than one (Figure 4). The NCDS provided the data parameters that define the "safe" boundaries of dialysis. The kinetic modeling is the 


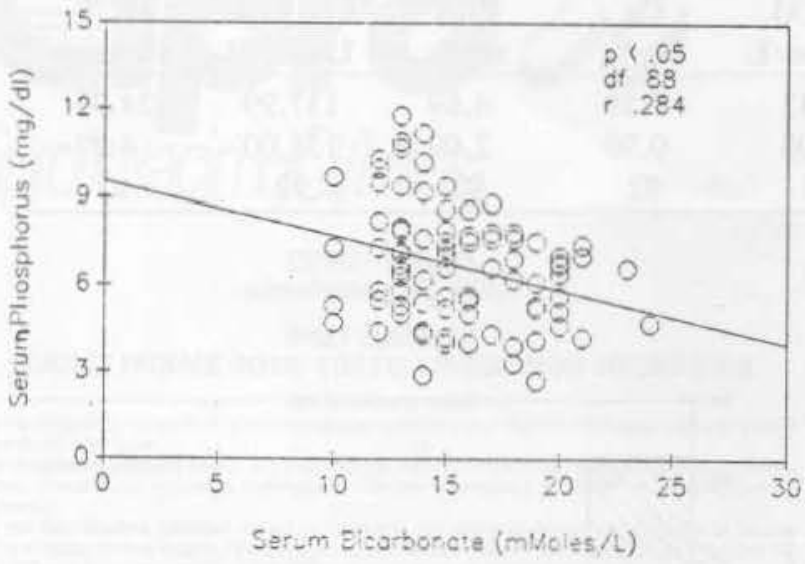

Figure 3. Regression analysis of serum phosphorus and serum bicarbonate in chronic dialysis patients.

vehicle that assures that the prescription for hemodialysis remains within those boundaries. To shorten dialysis time without prescription may be harmful.

\section{Recombinant human erythropoeitin therapy}

The development of normocytic anemia in most patients with chronic renal failure impairs the success of maintenance dialysis therapy. Anemia can be a complication of the hemodialysis procedure itself, with its associated blood losses and mild effect on oxygen transport functioning. The most important mechanism leading to anemia involves the production of subnormal levels of erythropeoitin. Other factors such as iron deficiency anemia, hyperparathyroidism, systemic infections, and aluminum toxicity may contribute to anemia in some patients. Increased hemolysis may also play a role in the anemia of chronic renal failure. There are several established treatment methods for anemia: transfusions; supplemental iron, vitamin B12, or folic acid when indicated; a change to peritoneal dialysis; parathyroidectomy; and the administration of androgens. None of these treatments have yielded satisfactory results, and some, such as transfusions and androgen therapy, pose risks and have serious side effects. A comparatively new approach, administration of genetically engineered erythropoeitin (R-HuEPO; EPOGEN, AMJGEN Inc.), has been found effective in treating anemia in clinical trials. Patients have shown improved cardiac performance as well as enhanced quality of life. Hypertension appears to be the most serious side effect of R-HuEPO therapy. ${ }^{*}$

A significant number of patients who are transfusion dependent with hemodialysis, benefit from the use of R-HuEPO. A recent report of the multicenter

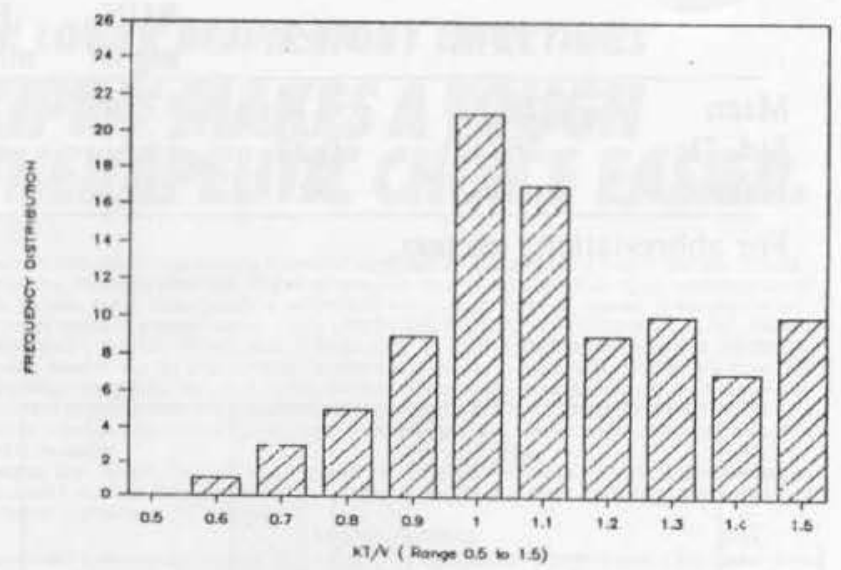

Figure 4. Frequency of distribution of $\mathrm{KT} / \mathrm{V}$ in chronic dialysis patients.

R-HuEPO by Eschbach involved 247 anemic hemodialysis patients who were treated with 150 to $300 \mathrm{U} / \mathrm{kg}$ of intravenous R-HuEPO three times a week for up to four monthss ${ }^{5}$ Of the 247 patients, 127 were deemed transfusion dependent prior to R-HuEPO therapy. Three months after the treatment with R-HuEPO, only three patients remained transfusion dependent. The risks associated with transfusions were thus largely eliminatd and the hematocrits were stabilized at acceptable levels. Because of the risks associated with transfusions, some patients having borderline hematocrit levels (i.e. $18 \%$ to $21 \%$ ) may resist undergoing transfusions in spite of significant anemia. With the availability of a safe mode of therapy these patients will achieve adequate hematocrit levels following the R-HuEPO therapy.

At doses of $50 \mathrm{U} / \mathrm{kg}$ or higher, dose dependent increases were consistently observed in reticulocyte count, erythron transferrin uptake, and rate of rise in hematocrit. Mean hematocrit level increased in these patients from $22.9 \%$ to $33.5 \%$; only five patients, most of whom had microcytosis, did not respond to treatment. Doses between 100 and $150 \mathrm{U} / \mathrm{kg}$ are sufficient to increase hematocrit levels to normal in two months or less. ${ }^{6}$ The starting do should range between $30-50 \mathrm{unit} / \mathrm{kg}$. The treatment does not induce neutralizing antibodies and produces few side effects; hypertension is the most common side effect but is easily controlled. There may be a tendency toward hyperkalemia and hyperphosphatemia, and there may be an increased heparin requirement to prevent clotting of the dialyzers. Occurrence of seizures and arterial thrombosis, including clotting of the vascular access, have been observed with the R-HuEPO therapy. 


\section{Rapid high-efficiency dialysis}

As compared to the conventional dialysis procedure, which takes about four hours, rapid higheffiency dialysis can accomplish the same results in two hours. To achieve this goal, solute removal, fluid removal and patient comfort must be maintained equal to or better than that delivered on standard acetate hemodialysis. High-efficiency hemodialysis therapies have been successfully applied using highclearance conventional and high-flux dialysis membranes. Urea indices $(\mathrm{KT} / \mathrm{V})$ have been used as a guide to prescribe therapy and reduce the risk of morbidity. These indices have ranged from 1.00 to 1.4. Typical dialyzer urea clearances are in the $265-340 \mathrm{ml} / \mathrm{min}$ range with blood flow rates averaging $350-400 \mathrm{ml} / \mathrm{min}$. The use of bicarbonate dialysate has been necessary to reduce the incidence of nausea, vomiting, headache and symptomatic hypotension during dialysis. Adequate cleaning and sterilization of bicarbonate concentrate contrainers and machines are required to prevent bacterial overgrowth and malfunction of ultrafiltration equipment. The issue of beta- 2 microglobulin removal by high flux dialysis is not fully resolved. (Elevated levels of this protein are known to cause carpel tunnel syndrome.)

The biochemical and hypertension control with high flux dialysis is the same as with the conventional dialysis and the response to R-HuEPO with these modalities of treatment is virtually identical. It is clear that continuing economic pressures related to reimbursement for dialysis may encourage the application of high-efficiency therapies in the future.

\section{Conclusion}

During the past five years there has been a marked change in management strategies for dialysis patients. More and more elderly patients and an increased number of diabetic patients with systemic complications are now being dialyzed. The two major long term complications, renal osteodystrophy and anemia, are now controlled better than before. The release of the R-HuEPO for clinical use will impact very favorably on patients' quality of life and the rehabilitation potential.

\section{References}

1. Van Stone JC: Hemodialysis, Chapter 10, Current Nephrology Vol 8. Edited by HC Gonick, Chicago: Year Book Medical Publishers Inc, 1985; pp 441-476.

2. Cushner HM, Adams ND: Review: Renal osteodystrophy-pathogenesis and treatment. Am J Med Sci 1986; 291: 264-75.

3. Lowrie EG. Teehan BP: Principles of prescribing dialysis therapy: Implementing recommendations from the NCDS. Kid Int 1983; 23 (suppl 13): 113-22.

4. Mohini R: Clinical efficacy of recombinant human erythropoeitin in hemodialysis patients. Seminars in Nephrology 1989; 9 (1, Suppl 1): 16-21.

5. Eschback JW, Adamson JW: Correction of anemia of hemodialysis (HD) patients with recombinant human erythropoeitin (R-HuEPO): Results of a multicenter study. Kidney Int 1988; 33:189 (abstr).

6. Eschbach JW, Egrie JC, Downing MR, et al: Correction of anemia of end stage renal disease with recombinant human erythropoeithin: Results of a combined phase I and phase II clinical trial. N Eng J Med 1987; 316: 73-78.

7. Collins AJ, Keshaviah P: Clinical application of high efficiency and high flux hemodialysis therapy. AKF Nephrology Letter 1988; 5(2): 21-25. 